\title{
Indwelling Tunneled Epidural Catheter for Analgesia in a Terminal Cancer Patient: A Case Report
}

\author{
Arunangshu Chakraborty*, Taniya Datta, Sanjit Agrawal \\ Tata Medical Center, India
}

Submission: June 24, 2017; Published: November 14, 2017

*Corresponding author: Arunangshu Chakraborty, Tata Medical Center, 14 MAR (EW), Newtown, Kolkata 700135, India, Tel: 9007733776; Email: drarunangshu@gmail.com

\begin{abstract}
Continuous epidural analgesia through tunneled epidural catheter is effective and economical for terminally ill cancer patients, in whom systemic medications alone cannot offer adequate pain relief. We describe a patient with severe leg pain from unrespectable endometrial carcinoma, in whom we successfully used a tunneled epidural catheter for long term pain relief. We describe a few modifications of the usual technique that would make the process safer for the operator and reduce risk of epidural catheter infection.
\end{abstract}

Keywords: Tunneled Epidural Catheter; Intractable Pain; Palliative Care

Abbreviations: NIBP: Non Invasive Blood Pressure; ECG: Electrocardiogram; PCEA: Patient Controlled Epidural Analgesia; VAS: Vital Signs and Pain Score

\section{Introduction}

Pain in terminal cancer patients may be refractory to systemic analgesics. Epidural analgesia is effective in these patients [1]. Indwelling epidural catheter can provide long term pain relief; however, there are risks of catheter related infection and dislodgement of the catheter [2]. Subcutaneous tunneled epidural catheter insertion provides more security to the epidural catheter against dislodgement and reduces risk of infections. It also provides an easy access for self- administration of drugs [3]. We present a patient suffering from intractable pain caused by locally advanced endometrial carcinoma, in which we used an indwelling tunneled epidural catheter for long term pain relief. We also describe a simple technical modification for epidural tunneling which is potentially safe for the operator and carries less risk of infection compared to the usual tunneling technique. A written informed consent for scientific publication was obtained from the patient.

\section{Case Report}

A 47 year old female, weighing $55 \mathrm{~kg}$, presented initially with per vaginal discharge and irregular menstrual cycles for which she had undergone total laparoscopic hysterectomy and bilateral salpingo oophorectomy at another center. She was later diagnosed at our institution with cancer of endometrium grade II with bilateral pelvic and Para aortic lymph node involvement. While waiting for a completion surgery, she developed pain in the right leg which was managed conservatively. She received 6 cycles of chemotherapy without adequate pain relief. CT scan revealed a large right pelvic mass (diameter $8.4 \mathrm{~cm}$ ) encasing vessels and muscles making it unrespectable. She was advised best supportive care [4]. Initially a buprenorphine patch was prescribed for pain control but she came back to the palliative clinic after a month with excruciating pain in the right hip and thigh with a pain score of 7/10 (Visual analog scale-VAS). She was prescribed pregabalin $75 \mathrm{mg}$, thiocholchicoside $4 \mathrm{mg}$ and paracetamol $650 \mathrm{mg}$ four times a day. Buprenorphine patch was withdrawn in view of recently developed liver functions derangement. On the next visit 15 days later, she still had pain, VAS $7 / 10$. She was given oral morphine $30 \mathrm{mg}$, naproxen $500 \mathrm{mg}$, drotaverine $40 \mathrm{mg}$ and pregabalin $75 \mathrm{mg}$, but pain did not resolve and instead increased to 8/10 with spikes of 10/10 intensity. It could be controlled only with rescue dose of intravenous morphine. Pain was affecting her movement and daily activities. Oral morphine dose was increased to $60 \mathrm{mg}$ and later switched to intravenous route but even this did not help. At this point she was referred to our pain clinic where a tunneled epidural catheter insertion was planned. On the scheduled date the patient was brought to the operation theater and pulse oximeter, non invasive blood pressure (NIBP) and electrocardiogram (ECG) monitor were attached. Intravenous midazolam $1 \mathrm{mg}$ along with fentanyl 50 mcg were administered for procedural sedation.

\section{Technique}

Patient was positioned right lateral with knees and neck flexed (fetal position). The field was prepared with $2.5 \%$ chlorhexidine in alcohol solution and draped with sterile sheets. Third lumbar 
vertebra was identified with a $\mathrm{C}$ arm fluoroscope with image intensification. A transverse incision was made at the level of L3-4 inters pace (Figures 1 \& 2). A $20 \mathrm{G}$ flexometallic epidural catheter was inserted through an $18 \mathrm{G}$ Tuohy needle by loss of resistance technique. The location of the epidural catheter was confirmed by fluoroscope, $5 \mathrm{~cm}$ of the catheter was left in the epidural space. A test dose of $2 \mathrm{ml} 0.25 \%$ levo-bupivacaine was administered, which was found to relieve the pain immediately.

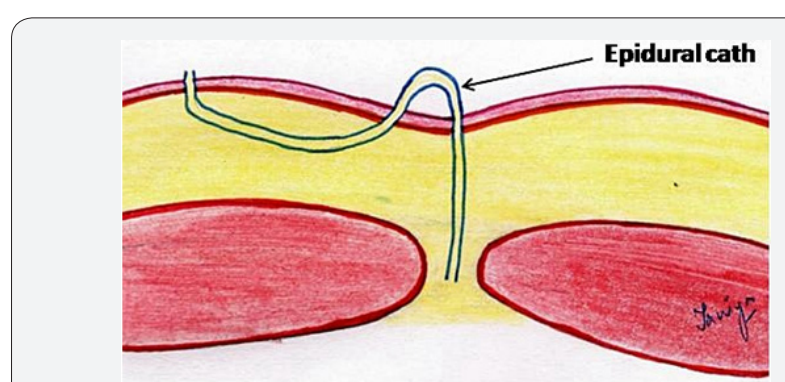

A

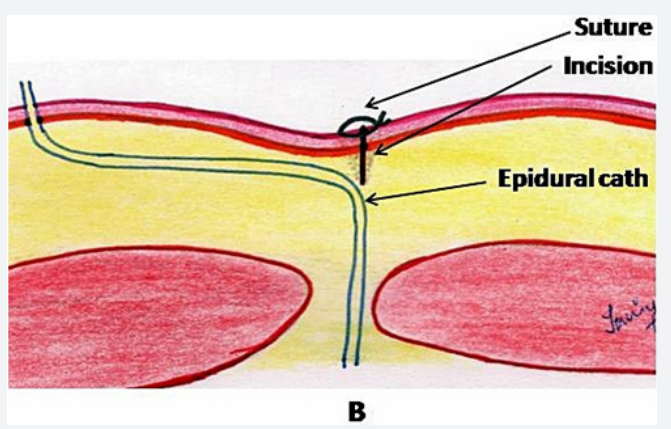

Figure 1: Schematic diagram showing the usual technique and our modification.

A. The usually practiced technique of epidural catheter tunneling: note that the epidural catheter comes out of the skin (arrow) and enters again into the tunnel to come out from the flanks.

B. Our modification: the incision (closed later with suture) allows complete tunneling of the epidural catheter.

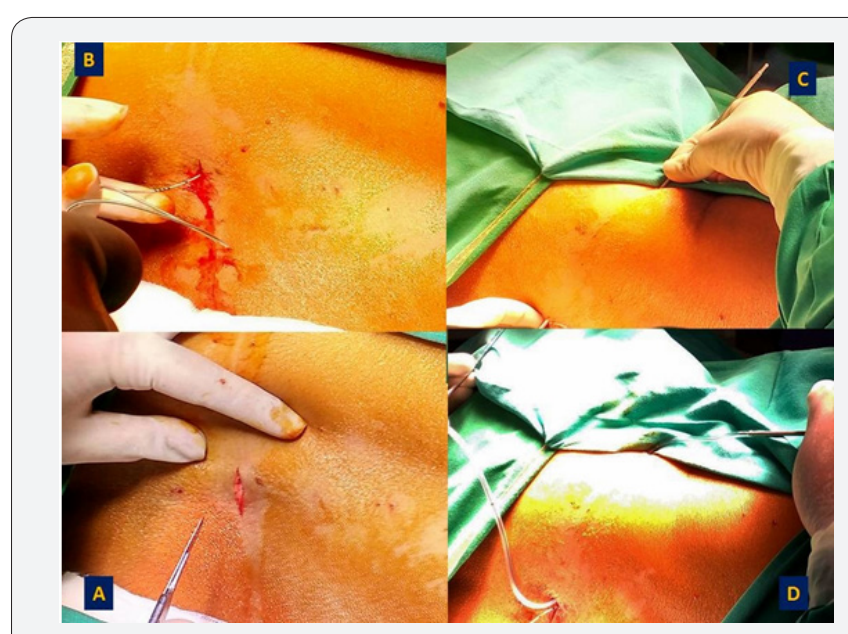

Figure 2 : Technique: Clockwise from left:

A. Skin Incision,

B. Epidural catheter has been inserted,

C. Blunt Tipped Chemoport Tunneler Used For Tunneling,

D. 12 Fr. Drain Catheter Attached To the Tunneler to Serve As a

Conduit for the Epidural Catheter.

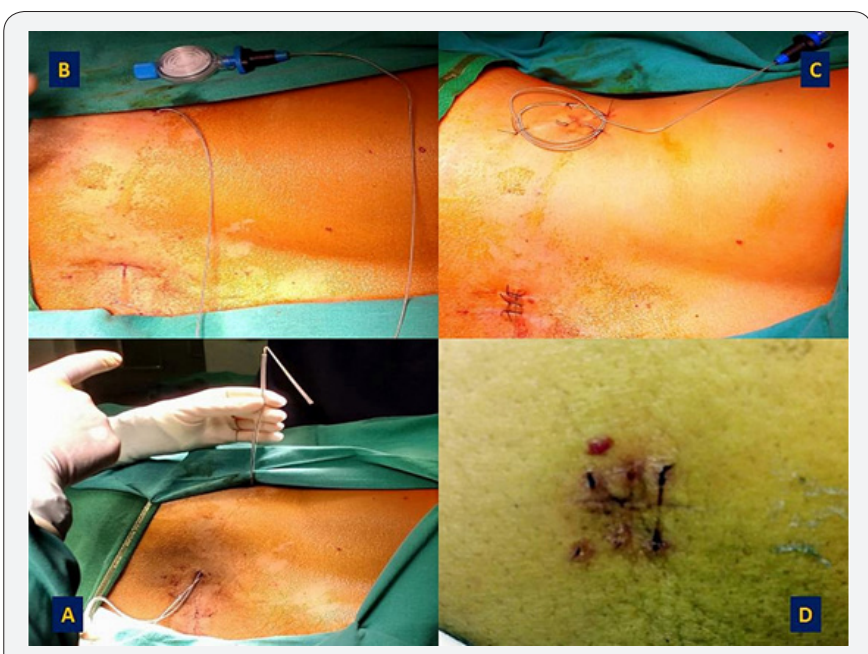

Figure 3 : Technique: Clockwise from left:

A. Epidural catheter has been passed through the drain tube,

B. Drain tube taken out to leave the epidural catheter in situ,

C. Skin incision sutured and the catheter anchored in the flank,

D. After 7 days- suture removed, wound healthy.

A blunt tipped malleable chemoport tunneler (Figures 2 \& 3) was inserted from the skin incision and used to make a subcutaneous tunnel to come out of the left flank. A 12 Fr drain catheter was attached to the proximal end of the tunneler and taken out from the other end. The tunneler was disengaged from the drain tube, epidural catheter inserted through the drain tube and the drain tube taken out, leaving only the epidural catheter in situ. The skin incision was sutured and transparent sterile adhesive dressing was applied. The epidural catheter was adequately anchored in the flank by sutures and sterile adhesive dressing. Another $1 \mathrm{ml}$ test dose was administered again to confirm smooth flow. A final fluoroscope image was taken to confirm position of the epidural catheter in situ. An epidural infusion of $0.1 \%$ levobupivacaine with $2 \mathrm{mcg} \mathrm{ml}-1$ fentanyl at the rate of $5 \mathrm{ml} / \mathrm{hr}$ was started. The patient was shifted to ward and monitored hourly for oxygen saturation, vital signs and pain score (VAS).

\section{Progress}

She reported a pain score of $0 / 10$ immediately after the procedure. In the ward, she reported a pain score less than $3 / 10$ consistently. She stayed in the hospital for seven days following the procedure, while she received other treatments for her illness. We visited her regularly and found her pain free. After 7 days the dressing and sutures at the epidural incision site were removed, wound had healed (Figure 3). The patient went home the next day with a patient controlled epidural analgesia (PCEA) pump. On the subsequent follow up visits for the next two months, there were no signs of infection or dislodgement of the catheter and she had lasting pain relief. The patient died after three months from the procedure.

\section{Discussion}

Pain relief is an important part of palliative care [5]. Majority of the patients are usually managed with oral and transdermal 
medications; many patients need an intervention to address pain and also lower the toxicity of high dose of opioids [6]. Neuraxial analgesia with a combination of opioid and local anaesthetic is one of the commonly used modalities of interventional pain management [7]. Well titrated epidural analgesia can allow the patients to be ambulant and carry on their activities of daily life independently, while controlling the pain adequately. The same can be achieved with an implantable intrathecal drug delivery system (IDDS) as well but the prohibitively high cost of IDDS restrict the scope of their use, particularly in developing economies. Continuous epidural infusion is preferred over IDDS for pain relief in cancer patients with lesser life expectancy as the former is more economical for a shorter period of use (3-6 months) [8].

Tunneling of the epidural catheter helps limit infection at the entry site, provides better anchorage over time and allows the epidural port to be self-accessed by the patient $[9,10]$. Various methods of tunneling have been described so far $[11,12]$, but none of them offer a complete tunneling as in the previously described techniques the epidural catheter used to come out of the skin and then again go through it into the tunnel at the point of epidural access (Figure 1). Thus a potential risk of infection stayed at the point of entry into epidural space. Also, by entering the tuohy needle from the flanks, there would be risks of needlestick injury to the operator and catheter shearing [13].

We did a few simple modifications of the technique: one, a transverse incision at the point of entry of the epidural needle, two, use of a blunt tipped chemoport tunneler for tunneling from the point of epidural access towards the flanks, three, using a 12 Fr drain catheter attached to the end of the tunneler, to serve as a conduit for the epidural catheter to exit from the tunnel. After the epidural catheter was inserted and passed through the tunnel, the incision was closed with sutures, thus effecting complete tunneling (Figures $1 \& 3$ ). We believe that our technique prevented any possibility of needle stick injury to the operator or shearing of the epidural catheter.

\section{Conclusion}

Continuous epidural analgesia through a tunneled epidural catheter can provide long lasting pain relief in terminally ill cancer patients with intractable pain. We believe that our modification of the technique makes the tunneling process safe and reduces chance of infection.

\section{References}

1. Yeon SJ, Jung AL, Jin WC, Eu GK, Hong SJ, et al. (2012) Efficacy of Epidural Analgesia in Patients with Cancer Pain: A Retrospective Observational Study. Yonsei Med J 53(3): 649-653.

2. Shaves M, Barnhill D, Bosscher J, Remmenga S, Hahn M, et al. (1991) Indwelling epidural catheters for pain control in gynecologic cancer patients. Obstet Gynecol 77(4): 642-644.

3. Carl P, Crawford ME, Ravlo O (1984) Fixation of extradural catheters by means of subcutaneous tissue tunnelling. Br J Anaeth 56(12): 13691371.

4. Mandaus L, Blomberg R, Hammar E (1982) Longterm epidural morphine analgesia. Acta Anaesthesiol Scand Suppl 74 149-150.

5. Nersesyan H, Slavin KV (2007) Current approach to cancer pain management: Availability and implications of different treatment options. Ther Clin Risk Manag 3(3): 381-400.

6. Rogers E, Mehta S, Shengelia R, Reid MC (2013) Four Strategies for Managing Opioid-Induced Side Effects in Older Adults. Clin Geriatr 21(4).

7. Farquhar Smith P, Chapman S (2012) Neuraxial (epidural and intrathecal) opioids for intractable pain. Br J Pain 6(1): 25-35.

8. Hawley P, Beddard Huber E, Grose C, McDonald W, Lobb D, et al. (2009) Intrathecal infusions for intractable cancer pain: A qualitative study of the impact on a case series of patients and caregivers. Pain Res Manag 14(5): 371-379.

9. De Jong PC, Kansen PJ (1994) A comparison of epidural catheters with or without subcutaneous injection ports for treatment of cancer pain. Anesth Analg 78(1): 94-100.

10. Heo BH, Pyeon TH, Lee HG, Kim WM, Choi JI, et al. (2014) Epidural Infusion of Morphine and Levobupivacaine through a Subcutaneous Port for Cancer Pain Management. Korean J Pain 27(2): 139-144.

11. Tripathi M, Pandey M (2000) Epidural catheter fixation: subcutaneous tunnelling with a loop to prevent displacement. Anaesthesia 55(11): 1113-1116.

12. Sharma A, Parasa SK, Tejvath K (2016) Ramachandran G Epidural catheter fixation. A comparison of subcutaneous tunneling versus device fixation technique. Journal of Anaesthesiology, Clinical Pharmacology 32(1): 65-68.

13. Tripathi M (2012) Safe practices in epidural catheter tunneling. J Anaesthesiol Clin Pharmacol 28(1): 138-139.

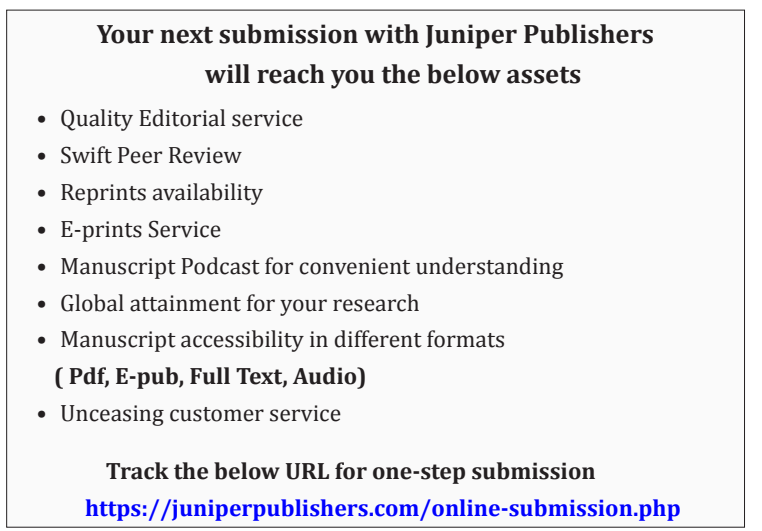

